\title{
Anterior nuclear deep brain stimulation guided by concordant hippocampal recording
}

\author{
Jamie J. Van Gompel, MD, ${ }^{1}$ Bryan T. Klassen, MD, ${ }^{2}$ Gregory A. Worrell, MD, PhD, ${ }^{2}$ \\ Kendall H. Lee, MD, PhD, ${ }^{1}$ Cheolsu Shin, MD, ${ }^{2}$ Cong Zhi Zhao, MD, ${ }^{2}$ Desmond A. Brown, MD, PhD, ${ }^{1}$ \\ Steven J. Goerss, ${ }^{1}$ Bruce A. Kall, MS, ${ }^{1}$ and Matt Stead, MD, PhD ${ }^{2}$
}

Departments of ${ }^{1}$ Neurosurgery and ${ }^{2}$ Neurology, Mayo Clinic, Rochester, Minnesota

\begin{abstract}
OBJECT Anterior nuclear (AN) stimulation has been reported to reduce the frequency of seizures, in some cases dramatically; however, it has not been approved by the US Food and Drug Administration. The anterior nucleus is difficult to target because of its sequestered location, partially surrounded by the ventricle. It has traditionally been targeted by using transventricular or lateral transcortical routes. Here, the authors report a novel approach to targeting the anterior nucleus and neurophysiologically confirming effective stimulation of the target, namely evoked potentials in the hippocampus.
\end{abstract}

METHODS Bilateral AN 3389 electrodes were placed in a novel trajectory followed by bilateral hippocampal 3391 electrodes from a posterior trajectory. Each patient was implanted bilaterally with a Medtronic Activa PC+S device under an investigational device exemption approval. Placement was confirmed with CT. AN stimulation-induced hippocampal evoked potentials were measured to functionally confirm placement in the anterior nucleus.

RESULTS Two patients had implantations by way of a novel AN trajectory with concomitant hippocampal electrodes. There were no lead misplacements. Postoperative stimulation of the anterior nucleus with a PC+S device elicited evoked potentials in the hippocampus. Thus far, both patients have reported a $>50 \%$ improvement in seizure frequency.

CONCLUSIONS Placing AN electrodes posteriorly may provide a safer trajectory than that used for traditionally placed AN electrodes. In addition, with a novel battery that is capable of electroencephalographic recording, evoked potentials can be used to functionally assess the Papez circuit. This treatment paradigm may offer increased AN stimulation efficacy for medically intractable epilepsy by assessing functional placement more effectively and thus far has proven safe. Clinical trial registration no.: NCT02235792 (clinicaltrials.gov).

http://thejns.org/doi/abs/10.3171/2015.3.FOCUS1541

KEY WORDS epilepsy; anterior nucleus; hippocampus; neuromodulation

$\mathrm{T}$ HALAMIC anterior nuclear (AN) stimulation for patients with medically and often surgically refractory epilepsy seems to be a promising therapy for palliation of focal and multifocal epilepsy. ${ }^{5,7,9} \mathrm{~A}$ recent multiinstitutional study designed to assess the efficacy of AN stimulation for medically refractory epilepsy, known as the Stimulation of the Anterior Nucleus of the Thalamus for Epilepsy (SANTE) trial, found that at the primary end point of 3 months after randomization, the frequency of seizures was reduced in the stimulation group by $>50 \%$ in
$40 \%$ of the patients compared with $15 \%$ in the group of patients who did not receive AN stimulation. ${ }^{22}$ In addition, after 2 years, AN stimulation resulted in $54 \%$ of the patients having a reduction in seizure frequency of $>50 \%$, and $15 \%$ of the patients reported $\geq 6$ months of freedom from seizures. ${ }^{13}$ Despite these encouraging results, AN stimulation has not been approved by the US Food and Drug Administration for use for medically refractory epilepsy; however, it has been approved outside the United States.

In the SANTE trial, the rate of lead misplacement and

ABBREVIATIONS AC = anterior commissure; $\mathrm{AN}=$ anterior nuclear; $\mathrm{DBS}=$ deep brain stimulation; $\mathrm{EEG}=$ electroencephalographic; $\mathrm{MMT}=$ mammillothalamic tract; $\mathrm{MNI}=$ Montreal Neurological Institute; PC = posterior commissure; SANTE = Stimulation of the Anterior Nucleus of the Thalamus for Epilepsy.

SUBMITTED February 1, 2015. ACCEPTED March 11, 2015.

INCLUDE WHEN CITING DOI: 10.3171/2015.3.FOCUS1541.

DISCLOSURE All implanted components, including Activa PC+S devices, spine lead extensions, and intracranial electrodes (3389 and 3391 ), were provided by Medtronic as part of this trial. Drs. Van Gompel, Klassen, Worrell, Lee, and Stead report funded research with Medtronic; Drs. Shin, Zhao, Brown, Kall, and Goerss report no conflicts of interest concerning the materials or methods used in this study or the findings specified in this paper. 
revision was $8.2 \% .{ }^{22}$ It is unfortunate that, based on these results, the anterior nucleus seems to be a difficult target to reliably hit. Therefore, 2 techniques may aid in overcoming misplacement and perhaps enhancing the efficacy of AN stimulation. One technique is to take advantage of an alternative trajectory to the anterior nucleus to avoid the complications seen with the use of other trajectories; a second technique is to electrophysiologically confirm placement within the anterior nucleus by measuring evoked potentials within the Papez circuit. The study described in this paper demonstrated the culmination of these ideas.

\section{Methods}

This study was registered with the ClinicalTrials.gov database (http://clinicaltrials.gov), and its registration no. is NCT02235792 .

\section{Patient Selection}

We obtained a Food and Drug Administration investigational device exemption and Mayo Clinic institutional review board approval for the treatment of 5 patients with medically intractable epilepsy according to the protocol described here. We selected patients who 1) had drugresistant epilepsy, defined as failure of at least 3 appropriately used anticonvulsant medications, 2) were not surgical candidates based on video electroencephalographic (EEG) monitoring, 3) had bilateral independent temporal lobe seizures, and 4 ) had $\geq 2$ consciousness-impairing seizures per month for at least 3 months before enrollment. Each patient consented to this study after approval at a multidisciplinary epilepsy surgery conference that included epileptologists, neuroradiologists, neurosurgeons, and neuropsychologists. The patients were required to keep a seizure diary 3 months before implantation and to keep the same diary postoperatively. Here, we report the technique we developed and used.

\section{Surgical Implantation}

Each patient was placed under anesthesia, a Leksell (Elekta) frame was placed, and stereotactic MR images were obtained. These data were then used to identify the anterior commissure (AC)/posterior commissure (PC) with COMPASS (COMPASS International) deep brain stimulation (DBS)-targeting software. Using a Schaltenbrand and Wahren atlas overlay and anatomical guidances,$^{14}$ we targeted the anterior nucleus. Initially targeted were coordinates from Hodaie et al., ${ }^{6}$ and we used $6 \mathrm{~mm}$ from midline, $12 \mathrm{~mm}$ superior to the $\mathrm{AC} / \mathrm{PC}$, and $8 \mathrm{~mm}$ anterior to the PC as a starting point; however, we modified our target based on anatomy to allow contact 0 to be as high and medial in the anterior nucleus as possible, given that our trajectory was from inferior posterior to anterior superior, as opposed to classic transventricular targeting (Fig. 1). Intraoperative microelectrode recordings were not used because each patient was under general anesthesia; we used general anesthesia for fear of the patient having a generalized seizure in the operating theater. We then also planned bilateral hippocampal trajectories designed to span $2.5 \mathrm{~cm}$ of the hippocampus and $1 \mathrm{~cm}$ of the amygdala. We used this approach to the anterior nucleus because of the entry point of the hippocampal electrodes, and our discussion will outline the potential benefits of such an approach. Medtronic 3389 electrodes were then implanted in the anterior nucleus, and Medtronic 3391 electrodes were implanted into the long axis of the hippocampus. Intraoperative fluoroscopy was used to ensure their accurate placement. After placement of the leads, the patient was taken immediately (while still under anesthesia) for postplacement CT to confirm localization (Fig. 2). After the electrode locations were confirmed, the patient was taken back to the operating theater. The leads were then tunneled to their respective sides, meaning that ipsilateral AN Contacts 0-3 and ipsilateral hippocampal Leads 4-7 were brought to the same Medtronic Activa $\mathrm{PC}+\mathrm{S}$ device. Bilateral battery implantation was performed independently, and 60-cm pain lead (37087-60; Medtronic) extensions were used to enable channel isolation for recordings greater that that of the standard DBS extension. The patient was then taken to the intensive care unit to recover and dismissed on Postoperative Day 3. The day after the operation, the sensing capabilities of the device were interrogated, and the data were recorded.

\section{Evoked Potential Recording}

Each patient was brought back 2 weeks after surgery and admitted to the hospital, and video electroencephalography was performed while evoked potentials were measured and the device was programmed. To assess the hippocampal evoked responses from AN stimulation, monopolar stimulation was delivered to each of the 4 anterior nucleus electrode contacts by using a rate of $2 \mathrm{~Hz}$, a pulse width of $90 \mu \mathrm{sec}$, and an amplitude of $4 \mathrm{~V}$. Responses were recorded across the most distal and most proximal contacts on the hippocampal electrode. The recording was divided into epochs beginning at the onset of each stimulus artifact, and these epochs were averaged to yield the hippocampal evoked potential resulting from stimulation at that specific contact (Fig. 3).

To visualize the implanted contacts in relation to the anterior nucleus, a preoperative MR image was segmented using the FSL-FIRST algorithm..$^{13}$ The thalamic segment was then normalized to the thalamus of the Morel digital atlas (in Montreal Neurological Institute [MNI] space) using SPM8 (Statistical Parametric Mapping 8). ${ }^{8}$ The postoperative CT image was coregistered to the preoperative MR image, and the previously calculated normalization was applied. MNI coordinates for the centers of each electrode artifact were then identified from the normalized postoperative CT image and plotted on the Morel atlas to show their relationship to the anterior nucleus (Fig. 3).

\section{Results}

Two patients were implanted with bilateral AN DBS leads (3389) and bilateral hippocampal leads (3391). There were no lead misplacements, as confirmed with a postplacement CT image merged with the preoperative plan on a preoperative MR image (Fig. 2) and further confirmed on $3 \mathrm{D}$ volumetric reconstructions of the electrodes within the thalamus, as described in Methods. An impressive postoperative lesional effect with a reduction in the frequency 

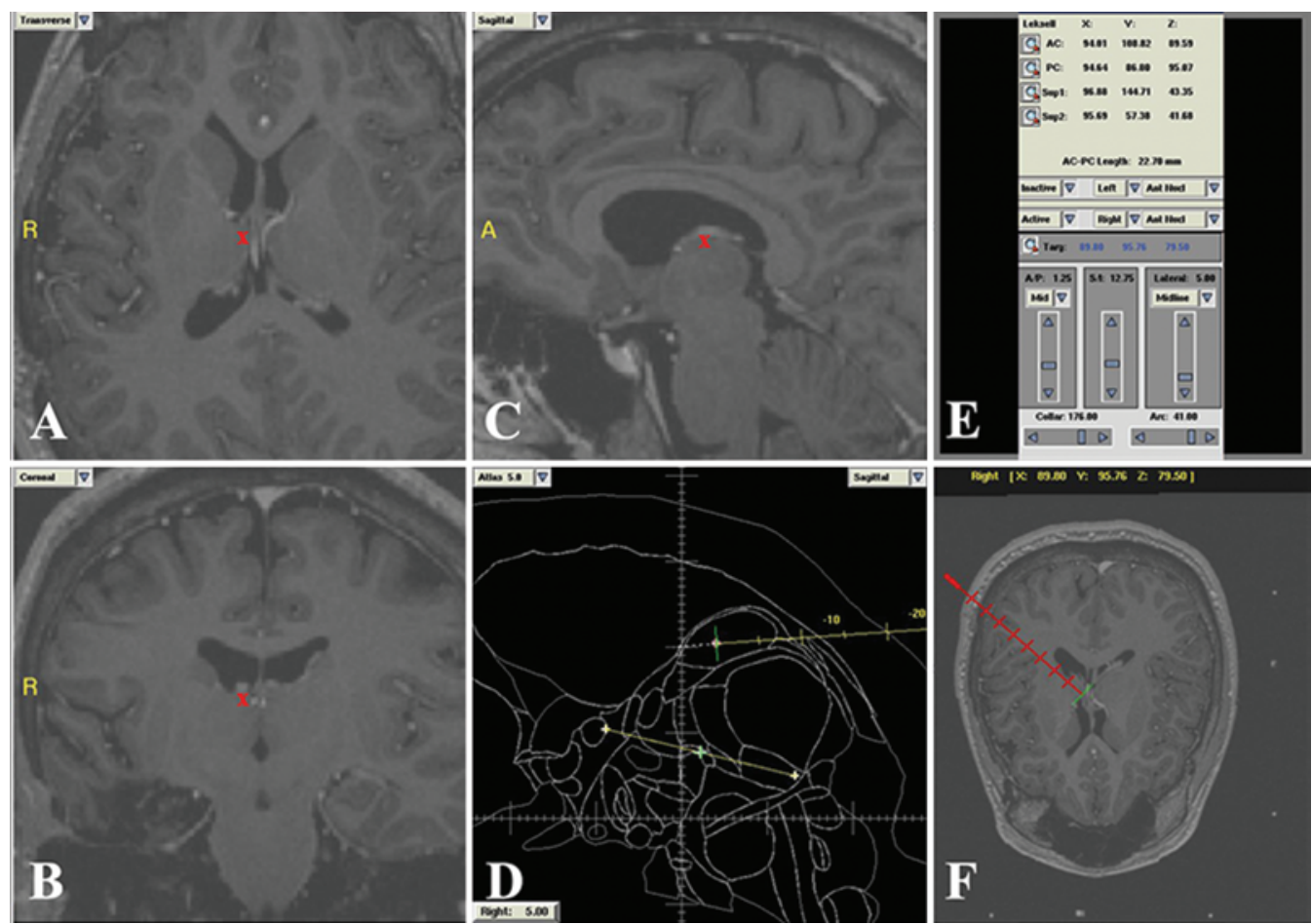

FIG. 1. AN trajectory: planning and placement. Shown are axial (A), coronal (B), and sagittal (C) MR images of the anatomically targeted anterior nucleus electrode. $\mathrm{R}=$ right; $\mathrm{A}=$ anterior; $\mathrm{x}(\mathrm{red})=$ target. $\mathrm{D}$ : Schaltenbrand and Wahren atlas representation $5 \mathrm{~mm}$ sagittal of the AN lead; placement was based on the AC/PC, and the target point is the center of the anterior nucleus. E: $\mathrm{X}, \mathrm{Y}, \mathrm{Z}$, arc, and collar coordinates for lead placement. F: Trajectory view of the planned electrode placement from a posterior trajectory.

of seizures was found in both of the patients. At the time of writing, these patients were doing well without complications. Monopolar stimulation of Contacts $0-3$ in all 4 anterior nucleus-positioned electrodes resulted in similar evoked potential patterns. Stimulation at Contacts 0 and 1 elicited well-formed hippocampal evoked potentials with negative peaks at approximately $40 \mathrm{msec}$ (Fig. 3), which matched the hippocampal evoked potentials previously reported in an ovine model of anterior nucleus stimulation using similar methodology. ${ }^{15}$ Stimulation at Contacts 2 and 3, both outside of the anterior nucleus according to the normalized atlas, yielded hippocampal potentials with a lower amplitude and greater latency $(60 \mu \mathrm{sec})$.

\section{Case 1}

This 26-year-old man had a 9-year history of complex partial seizures and simple partial seizures. He very rarely had secondary generalization. However, he typically had up to 5 simple partial seizures per week and a complex partial seizure once per month. In the first 2 postoperative weeks without stimulation, he had only 2 simple partial seizures. During his extended inpatient stay (for EEG monitoring and DBS programming), it was found that $4-\mathrm{Hz}$ stimulation at the bilateral AN leads led to improvements in his interictal electroencephalography results, and he was sent home with a setting for this low-frequency stimulation (Table 1). The hippocampal leads were used only to record. On his first outpatient return visit, he reported a significant reduction in his seizure frequency (only 9 seizures in the 6 weeks after his stimulation was activated). He left this programming session with both anterior nuclei set again at a low frequency of $7 \mathrm{~Hz}$ with 0.4 -second-on/0.1-second-off
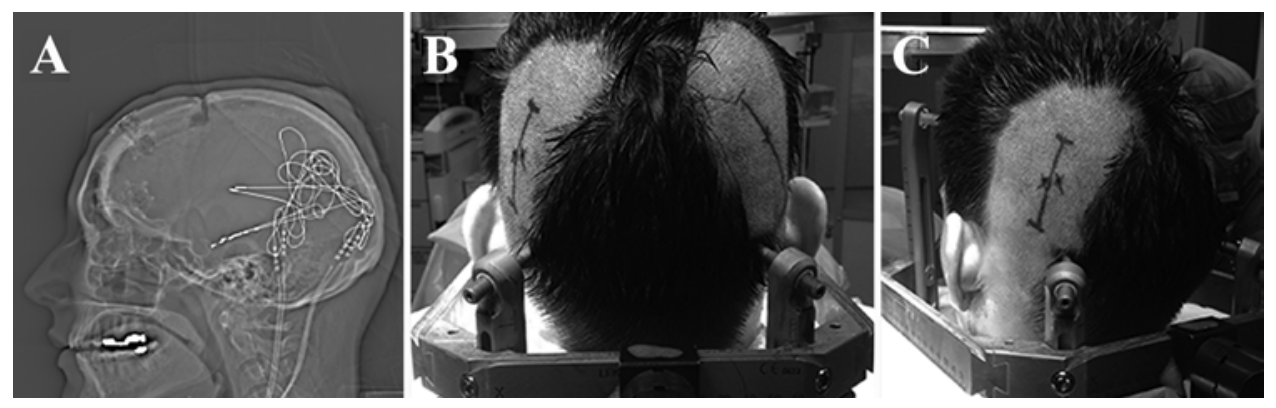

FIG. 2. Implantation. A: Postoperative CT scout film showing final implantation. B: Intraoperative incision planning. C: Implantation locations (note that the 2 small x's drawn on the skin are the planned entry sites for the hippocampal and AN leads). 


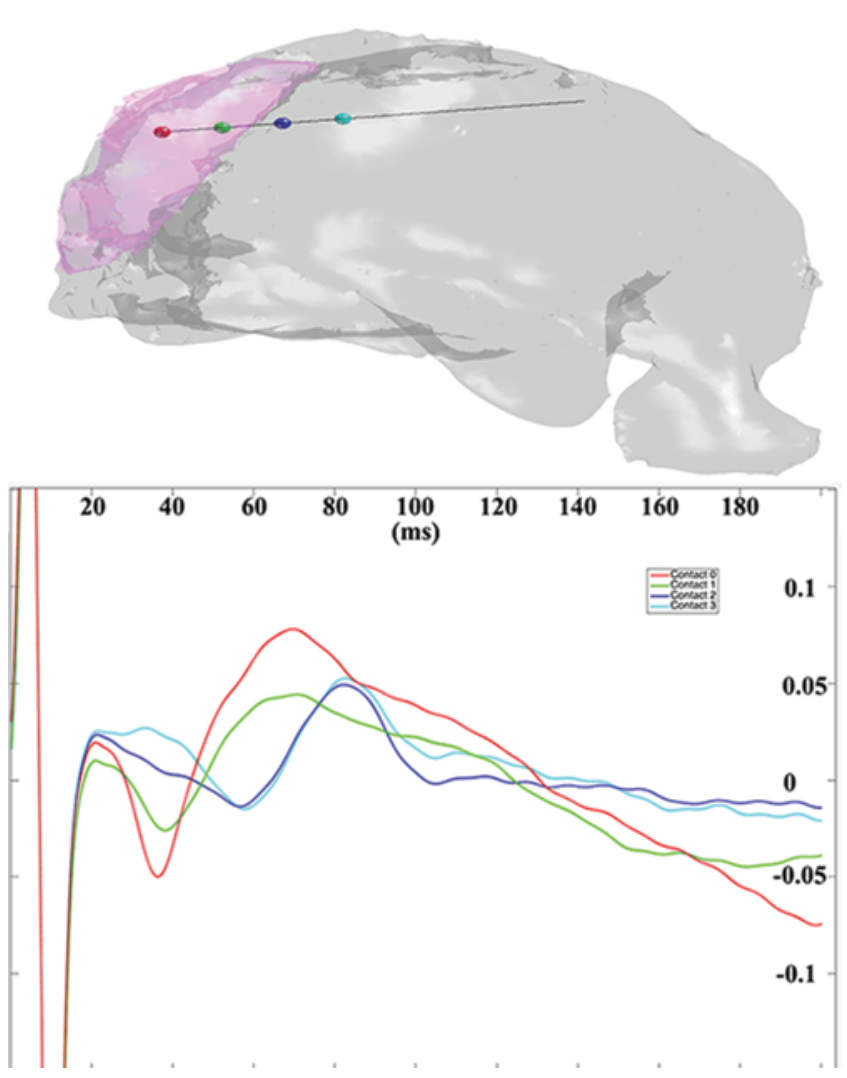

FIG. 3. Evoked potentials. Upper: Location of a right thalamic DBS electrode and its electrical contacts as translated into MNI coordinate space and superimposed on a 3D atlas. The area of the thalamus shaded magenta is the anterior nucleus, which fully contains the 2 most distal contacts. Lower: Evoked potentials elicited by stimulation at each of these contacts and recorded from the hippocampus; each trace is color matched to the contact as shown in the atlas. A well-formed evoked potential peaking at 50-60 msec was elicited with stimulation of the contacts inside the anterior nucleus, whereas stimulation at the other contacts elicited a smaller response with a later peak.

cycling (Table 1). On his second return visit 6 weeks later, he reported only 3 partial seizures, which is a remarkable decrease in seizure frequency compared with his preoperative baseline frequency. He had no complaints of adverse effects and was even able to fly to Colorado to cheer on his favorite football team. Overall, in the first 12 weeks of his active stimulation, he had a total of only 12 seizures, which is an $80 \%$ decrease from the 12 weeks preceding his surgery, when he reported at least 60 seizures (as recorded in his seizure diary).

\section{Case 2}

This 32-year-old woman had had predominantly complex partial seizures, occasional simple partial seizures, and secondary generalizations since her late teens. They occurred in clusters of 3-11 seizures every 2 weeks. At her admission for inpatient EEG monitoring and DBS programming, she reported no seizures in those 2 weeks without stimulation. Again, during her admission to the monitoring unit, the lower-frequency stimulation seemed to correlate with better EEG patterns, and she was sent home on 7-Hz stimulation without cycling in the bilateral AN leads and with the hippocampal leads only for recording (Table 1). After the admission and DBS activation, she was seizure free for almost 4 weeks and then experienced a return of 2 typical complex partial seizures around her usual time frame for seizures. She was seizure free for 3 weeks, after which she experienced another cluster of 3 complex partial seizures and 2 simple partial seizures. By her first outpatient visit she had gone another 5 weeks with only 1 complex partial seizure; however, she reported 2 episodes of intense dizziness without impaired consciousness for approximately 10 minutes, which she did not classify as her typical simple partial seizure, although she did feel that these episodes occurred in her typical seizure time frame. She subjectively described it as if she was on the brink of a seizure. Overall, in the first 12 weeks of active stimulation, she had 4 complex partial seizures and 3 simple partial seizures, a 53\% decrease from the 12 weeks before surgery, when she had 15 seizures. Other than the reported dizziness, she also has not had any adverse effects, and only slight changes were made to her programs (Table 1).

\section{Discussion}

Anterior nuclear stimulation has been demonstrated to suppress seizure occurrence, with a typical reported microlesional effect and 50\% reduction of seizure frequency 2 years after the initiation of stimulation. ${ }^{4} \mathrm{We}$ found similar seizure suppression in our 2 patients; however, it is still early, and stimulation typically reduces the frequency of seizures more prominently over time. Within the SANTE trial, a significant issue was lead misplacement and return to the operating theater for lead replacement $(8.2 \%$ of the cases). ${ }^{4}$ There has been some reluctance among surgeons who perform DBS to place AN electrodes transventricularly, because it may increase the risk of hemorrhage. However, traditional lateral transcortical approaches often

TABLE 1. Individual programming parameters*

\begin{tabular}{|c|c|c|c|}
\hline $\begin{array}{l}\text { Programming } \\
\text { Parameter }\end{array}$ & $\begin{array}{l}\text { Final Program at Discharge From Inpatient } \\
\text { Monitoring Unit }\end{array}$ & Final Program at 1st Postop Visit & Final Program at 2nd Postop Visit \\
\hline Patient 1 & $\begin{array}{l}\text { Contacts }-0,-1,+2 ; 90-\mu \text { sec pulse width; } \\
4-\mathrm{Hz} \text { frequency; 3.5-V amplitude; no } \\
\text { cycling }\end{array}$ & $\begin{array}{l}\text { Contacts }-0,-1,+2 ; 90-\mu \text { sec pulse width; } \\
\text { 7-Hz frequency; 3.5-V amplitude; } \\
\text { 0.4-sec-on/0.1-sec-off cycling }\end{array}$ & $\begin{array}{l}\text { Contacts }-0,-1,+2 ; 90-\mu \text { sec pulse width; } \\
\text { 7-Hz frequency; } 4.0-\mathrm{V} \text { amplitude; } \\
\text { 0.4-sec-on/0.1-sec-off cycling }\end{array}$ \\
\hline Patient 2 & $\begin{array}{l}\text { Contacts }-0,-1,+2 ; 90-\mu \text { sec pulse width; } \\
\text { 7-Hz frequency; 3.5-V amplitude; no } \\
\text { cycling }\end{array}$ & $\begin{array}{l}\text { Contacts }-0,-1,+2 ; 90-\mu s e c \text { pulse width; } \\
\text { 8-Hz frequency; 4.0-V amplitude; no } \\
\text { cycling }\end{array}$ & $\begin{array}{l}\text { Contacts }-0,-1,+2 ; 90-\mu \text { sed pulse width; } \\
\text { 8-Hz frequency; 4.0-V amplitude; no } \\
\text { cycling }\end{array}$ \\
\hline
\end{tabular}

\footnotetext{
* In each final program, both anterior nuclei had the same settings (only the hippocampal leads were recorded).
} 
transit critical cortex such as the operculum. Therefore, a more common approach for a neurosurgeon, albeit uncommon for those performing DBS, is the posterior inferior parietal approach used for shunts and biopsies. This approach for DBS placement may have less potential risk for an intraventricular bleed, because the electrode does not cross the ependyma twice. The risk of eloquent cortex injury with lateral transcortical approaches that use the operculum is also avoided by a more posterior trajectory. However, as reported previously, traditional targeting must be adjusted to anatomical targeting and the trajectory.

Another way to confirm proper electrode placement is neurophysiological confirmation by using stimulationinduced hippocampal evoked potentials. In this study, to ensure proper electrode placement, patients were readmitted for Medtronic Activa $\mathrm{PC}+\mathrm{S}$ system programing, and by stimulating the anterior nucleus and recording hippocampal evoked responses, we were reassured of the proper positioning (Fig. 3). This process also enables one to assess the most effective contacts for stimulation. Whereas the potentials reported here were recorded postoperatively, similar potentials were recorded in anesthetized sheep during AN electrode placement itself. ${ }^{15}$ Such an approach may be useful for fine-tuning the position of an electrode during the surgery itself.

In the past, evoked potential recordings required depth electrode placement and extraoperative stimulation; however, with this new sensing device (the Activa $\mathrm{PC}+\mathrm{S}$ ), one can avoid a second operation and have the ability to obtain recordings from the hippocampus regularly, which may confer an advantage. ${ }^{23-25}$ For example, stimulating parameters that elicit afterdischarges or other epileptiform patterns in the hippocampus are likely to be counterproductive and can be identified or avoided more easily with interval hippocampal monitoring. Also, as more is learned about the beneficial network effects of AN stimulation, it is conceivable that an electrophysiological biomarker for effective stimulation might be discovered, which could be useful for optimization of AN-stimulating parameters.

Anterior nucleus targeting is augmented by identification of the mammillothalamic tract (MMT), which runs parallel to transventricular targeting. Although we assume that AN stimulation is a key to seizure improvement in this strategy, it is possible that the varied effects of AN stimulation discussed in the literature relate to a failure to stimulate the MMT. It is interesting to note that there are sound animal data to indicate that ablation of the MMT, not the anterior nucleus, provides a treatment advantage in medically induced seizures. ${ }^{12}$ Therefore, a separate advantage of posterior approach targeting, as proposed in this paper, is placement of the lead perpendicular to the MMT. Being perpendicular to the MMT may increase the likelihood that the stimulation lead crosses the tract and further enable electric stimulation of the MMT; parallel approaches are more likely to miss the tract, especially when anatomical targeting of the MMT intentionally avoids this tract. It should be noted that parietal entry is a familiar concept to most neurosurgeons (as it relates to shunts and biopsies), so the complications one may see in this region of the brain are familiar. One particular issue that needs to be accounted for with this technique is that the wire loops typically have to be placed more inferior and anterior behind the ear, because the entry site for these leads is typically where they also rest. This lead location does not seem to result in any separate cosmetic outcome, and patients have not noted any additional discomfort.

Thus far in this study, we have used only AN stimulation and had excellent results. However, not only can the hippocampal leads be used for recording, but the patient may in fact receive stimulation to augment his or her seizures if additional intervention is needed. Hippocampal stimulation alone has shown additional efficacy for the control of focal medically refractory epilepsy. ${ }^{1-3,10,11,16-22}$ It is not yet known if combined AN and hippocampal stimulation would be more effective than stimulation at either site alone, and it is not clear how to best combine the stimulating parameters for the 2 targets.

\section{Conclusions}

Anterior nucleus targeting can be performed with an alternative trajectory that may be more amenable to proper AN placement with fewer adverse effects, although study involving more patients will be needed to prove this. In addition, postimplantation evoked response in the hippocampus from AN stimulation can be used to demonstrate functionally AN electrode placement, which may provide a reliable neurophysiological approach for targeting the nodes in the circuit of Papez.

\section{Acknowledgments}

We thank Karla K. Crockett, Kate Flicker, and Deb Gorman for their work with these patients and their tireless efforts for our institutional review board.

\section{References}

1. Boëx C, Seeck M, Vulliémoz S, Rossetti AO, Staedler C, Spinelli L, et al: Chronic deep brain stimulation in mesial temporal lobe epilepsy. Seizure 20:485-490, 2011

2. Bondallaz P, Boëx C, Rossetti AO, Foletti G, Spinelli L, Vulliemoz S, et al: Electrode location and clinical outcome in hippocampal electrical stimulation for mesial temporal lobe epilepsy. Seizure 22:390-395, 2013

3. Cukiert A, Cukiert CM, Argentoni-Baldochi M, Baise C, Forster CR, Mello VA, et al: Intraoperative neurophysiological responses in epileptic patients submitted to hippocampal and thalamic deep brain stimulation. Seizure 20:748-753, 2011

4. Fisher R, Salanova V, Witt T, Worth R, Henry T, Gross R, et al: Electrical stimulation of the anterior nucleus of thalamus for treatment of refractory epilepsy. Epilepsia 51:899-908, 2010

5. Graves NM, Fisher RS: Neurostimulation for epilepsy, including a pilot study of anterior nucleus stimulation. Clin Neurosurg 52:127-134, 2005

6. Hodaie M, Wennberg RA, Dostrovsky JO, Lozano AM: Chronic anterior thalamus stimulation for intractable epilepsy. Epilepsia 43:603-608, 2002

7. Kerrigan JF, Litt B, Fisher RS, Cranstoun S, French JA, Blum $\mathrm{DE}$, et al: Electrical stimulation of the anterior nucleus of the thalamus for the treatment of intractable epilepsy. Epilepsia 45:346-354, 2004

8. Krauth A, Blanc R, Poveda A, Jeanmonod D, Morel A, Székely G: A mean three-dimensional atlas of the human thalamus: generation from multiple histological data. Neuroimage 49:2053-2062, 2010 
9. Lim SN, Lee ST, Tsai YT, Chen IA, Tu PH, Chen JL, et al: Electrical stimulation of the anterior nucleus of the thalamus for intractable epilepsy: a long-term follow-up study. Epilepsia 48:342-347, 2007

10. Lockman J, Fisher RS: Therapeutic brain stimulation for epilepsy. Neurol Clin 27:1031-1040, 2009

11. Miatton M, Van Roost D, Thiery E, Carrette E, Van Dycke A, Vonck K, et al: The cognitive effects of amygdalohippocampal deep brain stimulation in patients with temporal lobe epilepsy. Epilepsy Behav 22:759-764, 2011

12. Mirski MA, Ferrendelli JA: Interruption of the mammillothalamic tract prevents seizures in guinea pigs. Science 226:72-74, 1984

13. Patenaude B, Smith SM, Kennedy DN, Jenkinson M: A Bayesian model of shape and appearance for subcortical brain segmentation. Neuroimage 56:907-922, 2011

14. Schaltenbrand G, Wahren W: Atlas for Stereotaxy of the Human Brain with Guide to the Atlas for Stereotaxy of the Human Brain, ed 2. Stuttgart: Thieme, 1977

15. Stypulkowski PH, Stanslaski SR, Denison TJ, Giftakis JE: Chronic evaluation of a clinical system for deep brain stimulation and recording of neural network activity. Stereotact Funct Neurosurg 91:220-232, 2013

16. Tyrand R, Seeck M, Spinelli L, Pralong E, Vulliémoz S, Foletti G, et al: Effects of amygdala-hippocampal stimulation on interictal epileptic discharges. Epilepsy Res 99:87-93, 2012

17. Velasco AL, Velasco F, Velasco M, Jiménez F, Carrillo-Ruiz JD, Castro G: The role of neuromodulation of the hippocampus in the treatment of intractable complex partial seizures of the temporal lobe. Acta Neurochir Suppl 97:329-332, 2007

18. Velasco AL, Velasco M, Velasco F, Menes D, Gordon F, Rocha L, et al: Subacute and chronic electrical stimulation of the hippocampus on intractable temporal lobe seizures: preliminary report. Arch Med Res 31:316-328, 2000

19. Velasco F, Velasco M, Velasco AL, Menez D, Rocha L: Electrical stimulation for epilepsy: stimulation of hippocampal foci. Stereotact Funct Neurosurg 77:223-227, 2001

20. Velasco M, Velasco F, Velasco AL: Centromedian-thalamic and hippocampal electrical stimulation for the control of intractable epileptic seizures. J Clin Neurophysiol 18:495513,2001

21. Velasco M, Velasco F, Velasco AL, Boleaga B, Jimenez F, Brito F, et al: Subacute electrical stimulation of the hippocampus blocks intractable temporal lobe seizures and paroxysmal EEG activities. Epilepsia 41:158-169, 2000

22. Vonck K, Boon P, Achten E, De Reuck J, Caemaert J: Longterm amygdalohippocampal stimulation for refractory temporal lobe epilepsy. Ann Neurol 52:556-565, 2002

23. Zumsteg D, Lozano AM, Wennberg RA: Depth electrode recorded cerebral responses with deep brain stimulation of the anterior thalamus for epilepsy. Clin Neurophysiol 117:1602-1609, 2006

24. Zumsteg D, Lozano AM, Wennberg RA: Mesial temporal inhibition in a patient with deep brain stimulation of the anterior thalamus for epilepsy. Epilepsia 47:1958-1962, 2006

25. Zumsteg D, Lozano AM, Wennberg RA: Rhythmic cortical EEG synchronization with low frequency stimulation of the anterior and medial thalamus for epilepsy. Clin Neurophysiol 117:2272-2278, 2006

\section{Author Contributions}

Conception and design: Van Gompel, Klassen, Worrell, Lee, Stead. Acquisition of data: Van Gompel, Klassen, Worrell, Zhao, Stead. Analysis and interpretation of data: all authors. Drafting the article: all authors. Critically revising the article: all authors. Reviewed submitted version of manuscript: all authors. Approved the final version of the manuscript on behalf of all authors: Van Gompel. Statistical analysis: Stead. Administrative/technical/ material support: Kall. Study supervision: Van Gompel, Klassen, Stead.

\section{Correspondence}

Jamie J. Van Gompel, Department of Neurosurgery, Mayo Clinic, 200 First St. SW, Rochester, MN 55905. email: vangompel. jamie@mayo.edu. 